\title{
SOME BOUNDARY VALUE PROBLEMS AND MODELS FOR COUPLED ELASTIC BODIES
}

\author{
$\mathrm{BY}$ \\ J. A. ARAngo (Departamento de Matemáticas, Universidad del Valle, Cali, Colombia), \\ L. P. LEBEDEV (Department of Mathematics and Mechanics, Rostov State University, \\ Rostov-on-Don, Russia), \\ AND \\ I. I. VOROVICH (Rostov State University, Rostov-on-Don, Russia)
}

Resumen. A new class of boundary value problems is presented. These problems are described by related equations of different nature and possess such properties as the appearance of highest derivatives in boundary conditions. Such problems appear to model common engineering constructions composed of elements of different mechanical natures like plates, shells, membranes, or three-dimensional elastic bodies.

Two problems are considered in detail, namely a three-dimensional elastic body with flat elements taken as a plate or a membrane, and a plate-membrane system. The existence-uniqueness theorems for the corresponding boundary value problems are established and an application of a conforming FEM is justified.

1. Introduction. The purpose is to construct and analyze some models of systems of coupled bodies subjected to external forces. The bodies are supposed to be described by different mathematical models, mainly using different approximations of the linear theory of elasticity. We consider in detail a Kirchhoff elastic plate coupled with a membrane or a plate with a three-dimensional elastic body.

Our approach is based on a general principle of mechanics, the virtual work principle, whose advantage is a comparatively easy way to derive a full statement of the corresponding boundary value problem together with natural conditions on the boundaries of bodies including their contact part and to formulate the FEM equations. Nonetheless, in addition, we give the standard formulation of the corresponding boundary value problem. First this makes it easier to see the advantage of our method: In the classical theory of elliptic problems one is faced with a change of order of ellipticity across inner borders and the occurrence of derivatives of highest order in the boundary conditions, difficulties avoided by the virtual work principle. Second, in one of the considered problems, the boundary conditions are of an unusual nature, being boundary value problems

Received April 17, 1995.

1991 Mathematics Subject Classification. Primary 73K20, 73C35. 
themselves, an interesting fact which cannot be seen easily within the framework of the virtual work principle.

In what follows, the summation convention over repeated indices will be used; Latin indices range from 1 to 3 whereas Greek indices take values 1 and 2. A subscript $\alpha$ followed by a comma means the derivative with respect to the corresponding $\alpha$-coordinate.

For static problems the virtual work principle is (see, for example, Truesdell [6])

$$
\delta W=\delta A \text {, }
$$

where $\delta W$ is the work of the internal forces on the virtual displacements $\delta \mathbf{u}$ (if the internal energy functional is determined then $\delta W$ is its first variation with respect to $\delta \mathbf{u}$ ).

To illustrate, let us consider elasticity in three dimensions. Here

$$
\delta W=\int_{V} \sigma_{i j}(\mathbf{u}) \delta \varepsilon_{i j}(\mathbf{u}) d V
$$

where $V$ is the volume occupied by the body, $\sigma_{i j}(\mathbf{u})$ is a component of stress tensor computed for a displacement vector $\mathbf{u}, \delta \varepsilon_{i j}(\mathbf{u})$ is the variation of $\varepsilon_{i j}(\mathbf{u}), \varepsilon_{i j}(\mathbf{u})$ being a component of the linear strain tensor

$$
\varepsilon_{i j}(\mathbf{u})=\frac{1}{2}\left(u_{i, j}+u_{j . i}\right)
$$

and thus

$$
\delta \varepsilon_{i j}(\mathbf{u})=\varepsilon_{i j}(\delta \mathbf{u})
$$

Now $\delta A$ is the work of external forces on the virtual displacements,

$$
\delta A=\int \mathbf{F} \cdot \delta \mathbf{u}
$$

so in this case the virtual work principle states

$$
\int_{V} \sigma_{i j}(\mathbf{u}) \delta \varepsilon_{i j}(\mathbf{u}) d V=\int \mathbf{F} \cdot \delta \mathbf{u}
$$

Depending on the nature of the problem, external forces may be of volume, surface, or line nature or combinations thereof.

We take Hooke's law in the form

$$
\sigma_{i j}(\mathbf{u})=C_{i j k l} \varepsilon_{k l}(\mathbf{u})
$$

where the moduli of elasticity $C_{i j k l}$ are supposed to have symmetry with respect to any pair of indices. Additionally, we assume that

$$
C_{i j k l} \varepsilon_{i j} \varepsilon_{k l}>\rho \varepsilon_{i j} \varepsilon_{i j}, \quad \text { with a constant } \rho>0 .
$$

In addition to smoothness conditions depending on the nature of the problem under consideration, the body displacement vector $\mathbf{u}$, as well as $\delta \mathbf{u}$, satisfy boundary conditions, part of which are imposed as geometrical constraints. For example, if a piece $S$ of the boundary $\partial V$ is kept fixed, we have

$$
\mathbf{u} \mid s=0
$$

and correspondingly for $\delta \mathbf{u}$

$$
\delta \mathbf{u} \mid s=0 .
$$


The other part of the boundary conditions is assumed to be natural.

As is well known ([6]), Eq. (1) considered for all admissible virtual displacements determines the equations of the corresponding boundary value problem as well as natural boundary conditions; vice versa, from a classical statement of the problem it is possible to obtain Eq. (1) and so, if a classical solution exists, if one adds a geometrical boundary condition of the type (4), Eq. (1) can be considered as equivalent to the statement of the problem. This brings us to the idea to use Eq. (1) to define a generalized solution of this mixed problem directly. Note that Korn's inequality (see [5]) allows us to use the symmetrical bilinear form $\delta W$ with respect to variables $\mathbf{u}$ and $\delta \mathbf{u}$ as a scalar product on a subspace of the Sobolev space $\left(\mathbf{H}^{1}(V)\right)^{3}$ of vector functions satisfying the boundary condition (4); so if we can show that $\delta A$ in (1) is a linear continuous functional with respect to $\delta \mathbf{u}$ on this subspace--and this is the case for a certain class of forces-then, using the Riesz representation theorem in Hilbert space, we get immediately a theorem of existence and uniqueness. Moreover, now we can write the equations of the Finite Element Method (FEM) for mechanical problems almost automatically.

Our point of view is that direct usage of the virtual work principle gives certain advantages in the study of complicated mechanical systems compared to the traditional approach.

- Natural boundary and natural contact conditions are obtained as a consequence of the principle; they agree with the nature of mechanical models of bodies, are correct, full and sometimes not self-evidently.

- Equation (1) can be used to formulate the equations of FEM directly instead of the long traditional way of multiplying the equation by virtual displacements, integrating and transforming the result of the same Eq. (1).

- Equation (1) gives in a natural way a generalized statement of the problem under consideration thereby dictating energy spaces for generalized solutions, a comparatively easy way to prove the theorems of existence and uniqueness of generalized solutions, and to justify the FEM.

We recall some basic facts about Sobolev spaces:

Let $m, n$ be nonnegative integers and $V$ a bounded domain in $\mathbf{R}^{n}$. Consider the internal product in $\mathbf{C}^{m}(\bar{V})$ :

$$
[u, v]=\sum_{|a| \leq m} \int_{V} D^{a} u(\mathbf{x}) D^{a} v(\mathbf{x}) d x_{1} \cdots d x_{n},
$$

$D^{a}$ being the partial derivative indicated by the multi-index $a=\left(a_{1}, \ldots, a_{n}\right)$ and $|a|=$ $a_{1}+\cdots+a_{n}$. The inherited norm is defined by

$$
\|u\|_{m, V}=[u, u]^{1 / 2} .
$$

The completion of $\mathbf{C}^{m}(\bar{V})$ with respect to the norm \|\|$_{m, V}$ is denoted by $\mathbf{H}^{m}(V)$. In $\mathbf{H}^{m}(V)$ we define the semi-norm

$$
|u|_{m, V}=\sum_{|a|=m} \int_{V} D^{a} u(\mathbf{x}) D^{a} u(\mathbf{x}) d x_{1} \cdots x_{n} .
$$

For the following results we refer the reader to [1]. 
TheOREM 1. If $V$ is bounded and $\partial V$ is regular, then there exists a constant $c>0$ such that

$$
\left(\int_{\partial V}\left|D^{a} u\right|^{2} d s\right)^{1 / 2} \leq c\left\|D^{a} u\right\|_{1, V}, \quad \forall u \in \mathbf{H}^{m}(V), \quad \forall a:|a| \leq m-1 .
$$

THEOREM 2. If $S$ is a smooth $n-1$ manifold in $\bar{V} \subset \mathbf{R}^{n}$ then

$$
\left(\int_{S} u^{2} d s+|u|_{1, V}^{2}\right) \text { and }\|u\|_{1, V}
$$

are equivalent norms in $\mathbf{H}^{1}(V)$.

Additionally, if $n=2\left(V \subset \mathbf{R}^{2}\right)$ and $S$ contains at least three points not lying on a straight line, then

$$
\left(\int_{S} u^{2} d s+|u|_{2, V}^{2}\right) \text { and }\|u\|_{2, V}
$$

are equivalent norms in $\mathbf{H}^{2}(V)$.

From the standard Sobolev embedding theorem we have the following:

Theorem 3. Let $V \subset \mathbf{R}^{n}$ be a bounded domain and let $\partial V$ be regular.

1. For $n=2$ the embedding operators

$$
\mathbf{H}^{m}(V) \rightarrow \mathbf{L}^{q}(V), \quad \mathbf{H}^{m}(V) \rightarrow \mathbf{L}^{q}(\partial V)
$$

for all $m \geq 1$ and all $q>1$, are continuous.

2. For $n=3$ the embedding operators

$$
\mathbf{H}^{1}(V) \rightarrow \mathbf{L}^{q}(V), \quad \mathbf{H}^{1}(V) \rightarrow \mathbf{L}^{p}(\partial V)
$$

for all $q \geq 6$ and all $p \geq 4$, are also continuous.

We further state some problems of applied mechanics concerning a system of two coupled bodies under load. The bodies are described by different mechanical models and, as a result, the functionals of external virtual work are the sums of two independent parts

$$
\delta W=\delta W_{1}+\delta W_{2}, \quad \delta A=\delta A_{1}+\delta A_{2},
$$

where the index $i$ refers to the $i$ th body. The bodies are related through the contact boundary; they have the same displacements on it, and there are stress relations on the contact boundary, a consequence of the virtual work principle.

2. Coupling of a plate and a membrane. First we consider a relatively simple problem for a plate coupled with a membrane; it is taken mainly to demonstrate our technique.

Choose a Cartesian coordinate system such that the middle surface of the plate, $\Omega_{p}$, and the region occupied by the membrane, $\Omega_{m}$, are placed on the $\left(x_{1}, x_{2}\right)$-plane. The region occupied by the plate is $\Omega_{p} \times\left[-\frac{h}{2}, \frac{h}{2}\right]$, where $h$ denotes the plate thickness. Assume that the contact line between the membrane and the plate $\Gamma$ is contained in the boundary $\partial \Omega_{p}$ of $\Omega_{p}$. 
In the case of a plate the virtual work done by the external forces on virtual (vertical) displacement $\delta w$ is

$$
\delta A_{p}=\int_{\Omega_{p}} F \delta w d x_{1} d x_{2}+\int_{\partial \Omega_{p}} f \delta w d s,
$$

where $F$ and $f$ depend on the volume and surfaces distributed forces acting on the plate.

We use the Kirchhoff model of the plate with the standard assumptions

$$
\sigma_{33}=0, \quad \varepsilon_{13}=\varepsilon_{23}=0 .
$$

Here we consider the bending problem,

$$
u_{\alpha}\left(x_{1}, x_{2}, x_{3}\right)=-x_{3} \frac{\partial w}{\partial x_{\alpha}}\left(x_{1}, x_{2}, 0\right),
$$

for all $\left(x_{1}, x_{2}\right) \in \Omega_{p}$ and all $-\frac{h}{2} \leq x_{3} \leq \frac{h}{2}$; thus

$$
\varepsilon_{\alpha \beta}(\mathbf{u})=-x_{3} \rho_{\alpha \beta}, \quad \varepsilon_{\alpha \beta}(\delta \mathbf{u})=-x_{3} \delta \rho_{\alpha \beta},
$$

where we use the standard notation for the change of curvature $\rho_{\alpha \beta}=\partial^{2} w / \partial x_{\alpha} \partial x_{\beta}$ and $\delta \rho_{\alpha \beta}=\partial^{2} \delta w / \partial x_{\alpha} \partial x_{\beta}$.

Kirchoff's hypothesis, together with (2), shows that the virtual work done by the internal forces is

$$
\delta W_{p}=\int_{\Omega_{p}} M_{\alpha \beta}(\mathbf{u}) \delta \rho_{\alpha \beta} d x_{1} d x_{2}
$$

where

$$
M_{\alpha \beta}(\mathbf{u})\left(x_{1}, x_{2}\right)=\int_{-\frac{h}{2}}^{\frac{h}{2}}-x_{3} \sigma_{\alpha \beta}(\mathbf{u}) d x_{3}, \quad \text { for all }\left(x_{1}, x_{2}\right) \in \Omega_{p}
$$

Now we derive an equivalent expression for $\delta W_{p}$ in Eq. (5). Note that using $\sigma_{33}=0$ and Hooke's law, $\varepsilon_{33}$ can be expressed in terms of $\varepsilon_{\alpha \beta}$; thus, all the stress components $\sigma_{\alpha \beta}$ can be expressed in terms of $\rho_{\alpha \beta}$. After some calculations we get

$$
\delta W_{p}=h^{3} \int_{\Omega_{p}} \widehat{C}_{\alpha \beta \gamma \theta} \rho_{\alpha \beta} \delta \rho_{\gamma \theta} d x_{1} d x_{2},
$$

where $\widehat{C}_{\alpha \beta \gamma \theta}$, depending on the moduli of elasticity, is symmetric with respect to different pairs of indexes, and, with regard to (3), we have

$$
\widehat{C}_{\alpha \beta \gamma \theta} \rho_{\alpha \beta} \delta \rho_{\gamma \theta}>\hat{\rho} \rho_{\alpha \beta} \delta \rho_{\alpha \beta},
$$

$\hat{\rho}$ being a positive constant.

On the other hand, for the membrane, the potential energy $\Phi$ as a functional of the displacement $w$ is known:

$$
\Phi(w)=\frac{k}{2} \int_{\Omega_{m}}\left[\left(\frac{\partial w}{\partial x_{1}}\right)^{2}+\left(\frac{\partial w}{\partial x_{2}}\right)^{2}\right] d x_{1} d x_{2}-\int_{\Omega_{m}} G w d x_{1} d x_{2}-\int_{\partial \Omega_{m}} g w d s,
$$

in which $G$ is a force, $g$ is line force, and $k$ is a constant depending on material and initial tension inside the membrane. The first integral in the expression of $\Phi(w)$ represents the 
internal energy of the membrane and therefore its first variation is

$$
\delta W_{m}=k \int_{\Omega_{m}}\left[\frac{\partial w}{\partial x_{1}} \frac{\partial \delta w}{\partial x_{1}}+\frac{\partial w}{\partial x_{2}} \frac{\partial \delta w}{\partial x_{2}}\right] d x_{1} d x_{2} .
$$

The other integrals give the work of external forces on virtual displacements $\delta w$,

$$
\delta A_{m}=\int_{\Omega_{m}} G \delta w d x_{1} d x_{2}+\int_{\partial \Omega_{m}} g \delta w d s .
$$

We assume that the displacements on the junction line are the same for the plate and the membrane:

$$
\left.w\right|_{\bar{\Omega}_{m}}(s)=\left.w\right|_{\bar{\Omega} p}(s) \text { for all } s \in \Gamma .
$$

Thus the classical solution of the coupled problem belongs to the class

$$
C_{1}=C\left(\overline{\Omega_{m} \cup \Omega_{p}}\right) \cap\left(C^{4}\left(\overline{\Omega_{p}}\right) \cup C^{2}\left(\overline{\Omega_{m}}\right)\right) .
$$

For $w, \delta w \in C_{1}$ we define

$$
\begin{gathered}
L(\delta w)=\delta A_{p}+\delta A_{m} \\
\langle w, \delta w\rangle_{m p}=\langle w, \delta w\rangle_{m}+\langle w, \delta w\rangle_{p}
\end{gathered}
$$

where $\langle w, \delta w\rangle_{m}=\delta W_{m}$ and $\langle w, \delta w\rangle_{p}=\delta W_{p}$.

Now we are coming to a decisive point: to the question of boundary conditions. Part of them are geometrical and part are of a mechanical nature. For definiteness we suppose that a connected piece $S \subset\left(\partial \Omega_{p} \cup \partial \Omega_{m} \cup \Gamma\right)$ is kept fixed; therefore, the geometrical constraint for $w$ is

$$
w=0 \text { on } S \text {. }
$$

Moreover, without loss of generality, we assume that $S_{1}=S \cap \partial \Omega_{p}$ contains at least three points that do not lie on the same straight line.

We know that the Kirchhoff plate model requires a second condition, say on $S_{1}$; it is possible to require

$$
\partial w / \partial n=0
$$

or to assign a moment.

As to the membrane, a condition of type 8 or 9 is needed. But what to do with a line of plate-shell contact? Each of the models requires a different number of boundary conditions and it is not at all clear how to choose them in a compatible way. One of them is evident: the displacements must be continuous and this was reflected above in the definition of $C_{1}$. Others are not so evident; for example, it is clear from the mechanical nature of the problem that we cannot require continuity of the derivatives. Our approach produces those conditions in a natural way.

One can show that $\langle,\rangle_{m p}$ is a bilinear form in $C_{1}$ satisfying $\langle w, \delta w\rangle_{m p}=\langle\delta w, w\rangle_{m p}$ and $\langle w, w\rangle_{m p} \geq 0$ for all $w, \delta w \in C_{1}$. Moreover, if $S$ contains three points of $\partial \Omega_{p}$ not belonging to a straight line then $\langle w, w\rangle_{m p}=0$ if and only if $w \equiv 0$. This means that $\langle,\rangle_{m p}$ is a scalar product in the linear space of functions $w \in C_{1}$ satisfying the geometrical constraint (8). 
Definition 4. The completion with respect to the norm $\|w\|_{m p}:=\left(\langle w, w\rangle_{m p}\right)^{1 / 2}$ of the subset of functions $C_{1}$ satisfying the geometrical constraint (8) is denoted by $\mathcal{H}$.

LEMMA 5. The norms

$$
\left(\|w\|_{2, \Omega_{p}}^{2}+\|w\|_{1, \Omega_{m}}^{2}\right)^{1 / 2} \quad \text { and } \quad\|w\|_{m p}:=\left(\langle w, w\rangle_{m p}\right)^{1 / 2}
$$

are equivalent in $\mathcal{H}$.

Proof. The inequality $\|w\|_{m p} \leq \operatorname{const}\left(\|w\|_{2, \Omega_{p}}^{2}+\|w\|_{1, \Omega_{m}}^{2}\right)^{1 / 2}$ is trivial.

Now note that the restriction of $w \in \mathcal{H}$ to $\Omega_{p}$ is an element of the space $\mathbf{H}^{2}\left(\Omega_{P}\right)$; so by virtue of Theorem 2 ,

$$
\|w\|_{2, \Omega_{p}}^{2} \leq \operatorname{const}\langle w, w\rangle_{p}
$$

By the same theorem, we have

$$
\|w\|_{1, \Omega_{m}}^{2} \leq \operatorname{const}\left(\langle w, w\rangle_{m}+\int_{\Gamma}|w|^{2} d s\right) .
$$

Taking into account the continuity condition (7), by Theorem 3 , we get

$$
\int_{\Gamma}|w|^{2} d s \leq \operatorname{const}\langle w, w\rangle_{p}
$$

Therefore, we have

$$
\|w\|_{2, \Omega_{p}}^{2}+\|w\|_{1, \Omega_{m}}^{2} \leq \operatorname{const}\left(\langle w, w\rangle_{p}+\langle w, w\rangle_{m}\right)
$$

which completes the proof.

The displacement problem for the plate-membrane system can be stated as a variational problem.

DEFINITION 6. $w \in \mathcal{H}$ is called a generalized solution of the plate-membrane boundary value problem with geometrical constraints (8) if it is a solution of the variational problem

$$
\langle w, \delta w\rangle_{m p}=L(\delta w), \quad \text { for all } \delta w \in \mathcal{H} \text {. }
$$

We need to put some restrictions on external forces. Let us assume that for some $q>1$

$$
G \in \mathbf{L}^{q}\left(\Omega_{m}\right), \quad g \in \mathbf{L}^{q}\left(\partial \Omega_{m}\right), \quad F \in \mathbf{L}^{1}\left(\Omega_{p}\right), \quad f \in \mathbf{L}^{1}\left(\partial \Omega_{p}\right) .
$$

In Definition (6) we said nothing about other boundary conditions. Since our approach is variational this means we imposed some conditions, which are called natural, implicitly. THEOREM 7. Under condition (11) the variational problem (10) has a unique solution $w \in \mathcal{H}$.

Proof. Consider the linear form $L$,

$$
\begin{gathered}
|L(\delta w)|=\mid \int_{\Omega_{m}} G \delta w d x_{1} d x_{2}+\int_{\partial \Omega_{m}} g \delta w d s+\int_{\Omega_{m}} d \delta w d s \\
+\int_{\Omega_{p}} F \delta w d x_{1} d x_{2}+\int_{\partial \Omega_{p}} f \delta w d s \mid .
\end{gathered}
$$


Applying the Hölder integral inequality, we get

$$
\begin{aligned}
|L(\delta w)| \leq & \left(\int_{\Omega_{m}}|G|^{q} d x_{1} d x_{2}\right)^{1 / q}\left(\int_{\Omega_{m}}|\delta w|^{p} d x_{1} d x_{2}\right)^{1 / p} \\
& +\left(\int_{\partial \Omega_{m}}|g|^{q} d s\right)^{1 / q}\left(\int_{\partial \Omega_{m}}|\delta w|^{p} d s\right)^{1 / p} \\
& +\left(\int_{\Omega_{p}}|F| d x_{1} d x_{2}\right) \max _{\Omega_{p}}|\delta w| \\
& +\left(\int_{\partial \Omega_{p}}|f| d s\right) \max _{\partial \Omega_{p}}|\delta w|, \quad 1 / q+1 / p=1
\end{aligned}
$$

and using the embedding Theorem 3 we see

$$
|L(\delta w)| \leq \operatorname{const}\left(\|\delta w\|_{2, \Omega_{p}}^{2}+\|\delta w\|_{1, \Omega_{m}}^{2}\right)^{1 / 2},
$$

and thus, by Lemma 5, we obtain

$$
|L(\delta w)| \leq \operatorname{const}\left(\|\delta w\|_{m, p}\right) .
$$

By the Riesz representation theorem, we therefore have

$$
L(\delta w)=\left\langle w_{0}, \delta w\right\rangle_{m p}
$$

where $w_{0} \in \mathcal{H}$ is the unique solution of the problem.

Now we state the classical formulation of the variational problem (10). To this end, regularity of $\delta w$ and $w$ is assumed. From the equation $\langle w, \delta w\rangle_{m p}=L(\delta w)$, we obtain for all $\delta w$,

$$
\begin{aligned}
\int_{\Omega_{p}} & M_{\alpha \beta} \delta w_{, \alpha \beta} d x_{1} d x_{2}+k \int_{\Omega_{m}} w_{, \alpha} \delta w_{, \alpha} d x_{1} d x_{2} \\
& -\int_{\Omega_{p}} F \delta w d x_{1} d x_{2}-\int_{\partial \Omega_{p}} f \delta w d s-\int_{\Omega_{m}} G \delta w d x_{1} d x_{2}-\int_{\partial \Omega_{m}} g \delta w d s=0 \\
& -\int_{\Omega_{m}}(k \Delta w+G) \delta w d x_{1} d x_{2}+\int_{\partial \Omega_{m}}(k \nabla w \cdot \mathbf{n}-g) \delta w d s \\
& +\int_{\Omega_{p}}\left(M_{\alpha \beta, \alpha \beta}-F\right) \delta w d x_{1} d x_{2}+\int_{\partial \Omega_{p}}\left(M_{\alpha \beta} \delta w_{, \alpha} n_{\beta}-M_{\alpha \beta, \beta} \delta w n_{\alpha}-f \delta w\right) d s=0
\end{aligned}
$$

where $\mathbf{n}=\left(n_{1}, n_{2}\right)$ are the components of the unitary normal vector to $\partial \Omega_{p}$. Putting $Q_{\alpha}=M_{\alpha \beta, \beta}$ and again integrating by parts, we get

$$
\begin{aligned}
\int_{\partial \Omega_{p}}\left(M_{\alpha \beta} \delta w_{, \alpha} n_{\beta}-M_{\alpha \beta, \beta} \delta w n_{\alpha}\right) d s & =\int_{\partial \Omega_{p}}(\nabla \delta w \cdot \mathbf{M} \cdot \mathbf{n}-\delta w \mathbf{Q} \cdot \mathbf{n}) d s \\
& =\int_{\partial \Omega_{p}}\left(M_{n n} \frac{\partial \delta w}{\partial n}-\left(\mathbf{Q} \cdot \mathbf{n}+\frac{\partial M_{n r}}{\partial s} \delta w\right)\right) d s
\end{aligned}
$$

where $M_{n n}=\mathbf{M} \cdot \mathbf{n} \cdot \mathbf{n}, M_{n r}=\mathbf{M} \cdot \mathbf{n} \cdot \tau$, and $\mathbf{M} \cdot \mathbf{n}=M_{\alpha \beta} n_{\alpha} \mathbf{e}_{\beta},\left\{\mathbf{e}_{1}, \mathbf{e}_{2}\right\}$ being the canonical basis of $\mathbf{R}^{2}$ and $\tau$ the unit tangent vector to $\partial \Omega_{p}$. Using standard techniques 
of variational analysis we get the following classical formulation of the couples problem under consideration:

$$
\begin{aligned}
& M_{\alpha \beta, \alpha \beta}-F=0 \text { in } \Omega_{p}, \\
& k \Delta w+G=0 \text { in } \Omega_{m}, \\
& M_{n n}=0 \text { on } \partial \Omega_{p}, \\
& \mathbf{Q} \cdot \mathbf{n}+\frac{\partial M_{n r}}{\partial s}+f=0 \text { on } \partial \Omega_{p} /(\Gamma \cup S), \\
& k \nabla w \cdot \mathbf{n}-g=0 \text { on } \partial \Omega_{m} /(\Gamma \cup S) \\
& \mathbf{Q} \cdot \mathbf{n}+\frac{\partial M_{n r}}{\partial s}+f=k \nabla w \cdot \mathbf{n}-g \quad \text { on } \Gamma / S .
\end{aligned}
$$

Equations (12) through (16) correspond to the classical theory of plates and membranes, whereas Eq. (17) describes the coupling of plate and membrane.

To complete the statement of the problem we need to add the geometrical condition $w=0$ on $S$.

As expected, on the contact line $\Gamma$ we have obtained an additional condition which is also natural; it means that the transversal force is continuous (the term $k \nabla w \cdot \mathbf{n}$ in the membrane theory has the mechanical meaning of transversal force too). We emphasize that on $\Gamma$ there are two additional conditions, the continuity condition

$$
\begin{gathered}
\left.w\right|_{\partial \Omega_{p} \cap \Gamma}=\left.w\right|_{\partial \Omega_{m} \cap \Gamma}, \\
\left.M_{n n}\right|_{\partial \Omega_{p} \cap \Gamma}=0,
\end{gathered}
$$

whose mechanical meaning is clear: the membrane cannot produce a moment.

Note that the boundary value problem considered is elliptic on both domains $\Omega_{m}$ and $\Omega_{p}$ with different order of ellipticity on the two domains.

3. Coupling of a plate and an elastic body. In this section we consider a plate and an elastic body under load which are patched together. This problem is more realistic than the one considered in the previous section; it occurs in different areas of applied mechanics. We assume that the normal of the plate remains normal to its mid-surface after loading and use the same Kirchhoff model as in Sec. 2. From the physical point of view it is clear that there are tangential deformations of the plate; thus, the displacement field $\mathbf{u}=\left(u_{1}, u_{2}, w\right)$ in the plate is given by

$$
\begin{gathered}
w\left(x_{1}, x_{2}-\frac{h}{2}\right)=w\left(x_{1}, x_{2}, 0\right), \\
u_{\alpha}\left(x_{1}, x_{2}, x_{3}\right)=u_{\alpha}\left(x_{1}, x_{2}, 0\right)-x_{3} \frac{\partial w}{\partial x_{\alpha}}\left(x_{1}, x_{2}, 0\right) .
\end{gathered}
$$

As in Sec. 2 we choose a Cartesian system in such a way that the middle surface of the plate $\Omega_{p}$ lies on the plane $x_{3}=0$. Let $V$ be the region occupied by the body. By $\Omega_{p b}$ we denote the lower panel of the plate $\left(x_{3}=-\frac{h}{2}\right)$ and suppose that $\Omega_{p b} \subset \partial V$. 
For the plate, the virtual work done by the external forces on the virtual displacement $\delta \mathbf{u}$ is given by

$$
\delta A p=\int_{\Omega_{p}} \mathbf{F} \cdot \delta \mathbf{u} d x_{1} d x_{2}+\int_{\partial \Omega p} \mathbf{F} \cdot \delta \mathbf{u} d s
$$

where $\mathbf{F}=\left(F_{1}, F_{2}, F\right)$ and $\mathbf{F}=\left(f_{1}, f_{2}, f\right)$ are obtained from the set of external forces (acting on the three-dimensional volume of the plate) using traditional tools of Kirchhoff plate theory. For the virtual work done by the internal forces we have

$$
\delta W_{p}=\int_{\Omega_{p}}\left(N_{\alpha \beta}(\mathbf{u}) \varepsilon_{\alpha \beta}(\delta \mathbf{u})+M_{\alpha \beta}(\mathbf{u}) \delta \rho_{\alpha \beta}\right) d x_{1} d x_{2}
$$

where $M_{\alpha \beta}(\mathbf{u})$ is defined as in Sec. 2 and

$$
N_{\alpha \beta}(\mathbf{u})=\int_{-h / 2}^{h / 2} \sigma_{\alpha \beta}(\mathbf{u}) d x_{3}
$$

it is seen that $\partial W_{p}$, as compared to $\delta W_{p}$ of Sec. 2 , has an additional term that counts for the tangential deformation of the plate.

Now let us model the second element of the couples problem the 3-dimensional elastic body. For definiteness, we again assume a piece of the body boundary $S$ to be kept fixed,

$$
\mathbf{u}=\mathbf{0} \text { on } S
$$

and forces given on the other part. For the body, the virtual work done by the external forces on a virtual displacement $\delta \mathbf{u}$ is given by

$$
\delta A_{b}=\int_{V} \mathbf{G} \cdot \delta \mathbf{u}+\int_{\partial V} \mathbf{g} \cdot \delta \mathbf{u} d s
$$

where $\mathbf{G}$ and $\mathbf{g}$ are the vectors of volume and surfaces forces respectively.

The virtual work $\delta W_{b}$ done by the internal forces is

$$
\delta W_{b}=\int_{V} \sigma_{i j}(\mathbf{u}) \delta \varepsilon_{i j}(\mathbf{u}) d x .
$$

Let us denote $\langle\mathbf{u}, \delta \mathbf{u}\rangle_{b}=\delta W_{b}$ and $\langle\mathbf{u}, \delta \mathbf{u}\rangle_{P}=\delta W_{p}$.

For external forces we assume that

$$
\begin{gathered}
F_{1}, F_{2} \in \mathbf{L}^{q}\left(\Omega_{p}\right), \quad F \in \mathbf{L}^{1}\left(\Omega_{p}\right), \quad f_{1}, f_{2} \in \mathbf{L}^{q}\left(\partial \Omega_{p}\right), \quad q>1, \\
G_{1}, G_{2}, G \in \mathbf{L}^{6 / 5}(V), \quad g_{1}, g_{2}, g \in \mathbf{L}^{4 / 3}(\partial V) .
\end{gathered}
$$

To be consistent with the notation of Sec. 2 we shall write

$$
\mathbf{u}= \begin{cases}\left(u_{1}, u_{2}, w\right) & \text { when }\left(x_{1}, x_{2}\right) \in \Omega_{p}, \\ \left(u_{1}, u_{2}, u_{3}\right) & \text { when }\left(x_{1}, x_{2}, x_{3}\right) \in V .\end{cases}
$$

Now we need to state some conditions on the contact surface. One of them is evident; this is the continuity of the displacement vector $\mathbf{u}=\left(u_{1}, u_{2}, w\right)$ on the contact region, namely, by the Kirchhoff hypothesis,

$$
w\left(x_{1}, x_{2},-\frac{h}{2}\right)=w\left(x_{1}, x_{2}, 0\right),
$$




$$
u_{\alpha}\left(x_{1}, x_{2},-\frac{h}{2}\right)=u_{\alpha}\left(x_{1}, x_{2}, 0\right)+\frac{h}{2} \frac{\partial w}{\partial x_{\alpha}}\left(x_{1}, x_{2}, 0\right), \quad \text { for all }\left(x_{1}, x_{2}\right) \in \Omega_{p},
$$

where $u_{1}\left(x_{1}, x_{2},-\frac{h}{2}\right), u_{2}\left(x_{1}, x_{2},-\frac{h}{2}\right)$, and $w\left(x_{1}, x_{2},-\frac{h}{2}\right)$ stand for the displacements of the body at the contact region with the plate, and the other terms refer to the plate.

We cannot require more smoothness of the displacement field on $\Omega_{p b}$; this would contradict the model of deformation. So we expect that our approach, using the Virtual Work Principle, will produce natural contact conditions.

A classical solution of the coupled problem has to belong to the class $C_{2}$ of vector functions $\mathbf{u} \in C^{2}(\bar{V}) \cup C^{4}\left(\overline{\Omega_{p}}\right)$ satisfying the conditions (18), (20), and (21).

As in Sec. 2, on $C_{2}$ we define

$$
A(\delta \mathbf{u})=\delta A_{p}+\delta A_{b}
$$

and

$$
\langle\mathbf{u}, \delta \mathbf{u}\rangle_{p b}=\langle\mathbf{u}, \delta \mathbf{u}\rangle_{P}+\langle\mathbf{u}, \delta \mathbf{u}\rangle_{b}
$$

It easily follows that $\langle\mathbf{u}, \delta \mathbf{u}\rangle_{p b}=\langle\delta \mathbf{u}, \mathbf{u}\rangle_{p b}$ and $\langle\mathbf{u}, \mathbf{u}\rangle_{p b} \geq 0$ for all $\mathbf{u} \in C_{2}$. Moreover, if $S \subset \partial V$ is a nondegenerate surface and $\mathbf{u}$ vanishes on $S$, then $\langle\mathbf{u}, \mathbf{u}\rangle_{p b}=0$ if and only if $\mathbf{u} \equiv 0$; this means that $\langle,\rangle_{p b}$ is a scalar product in the linear space $C_{2}$. We denote by $\mathcal{E}$ the completion of this linear space with respect to the norm $\|\mathbf{u}\|_{p b}:=\left(\langle\mathbf{u}, \mathbf{u}\rangle_{p b}\right)^{1 / 2}$.

LEMMA 8. In the space $\mathbf{H}^{1}\left(\Omega_{p}\right) \times \mathbf{H}^{1}\left(\Omega_{p}\right) \times \mathbf{H}^{2}\left(\Omega_{p}\right)$ the norms

$$
\left(\left\|u_{1}\right\|_{1, \Omega_{p}}^{2}+\left\|u_{2}\right\|_{1, \Omega_{p}}^{2}+\|w\|_{2, \Omega_{p}}^{2}\right)^{1 / 2}
$$

and

$$
\left(\int_{\Omega_{p}}\left(\left(u_{1}+\frac{h}{2} \frac{\partial w}{\partial x_{1}}\right)^{2}+\left(u_{2}+\frac{h}{2} \frac{\partial w}{\partial x_{2}}\right)^{2}+w^{2}\right) d x_{1} d x_{2}+\langle\mathbf{u}, \mathbf{u}\rangle_{P}\right)^{1 / 2}
$$

are equivalent.

Proof. We begin with the plane variant of Korn's inequality; in our notation it takes the form

$$
\left\|u_{1}\right\|_{1, \Omega_{p}}^{2}+\left\|u_{2}\right\|_{1, \Omega_{p}}^{2} \leq \int_{\Omega_{p}}\left(u_{1}^{2}+u_{2}^{2}\right) d x_{1} d x_{2}+\int_{\Omega_{p}} N_{\alpha \beta}(\mathbf{u}) \varepsilon_{\alpha \beta}(\mathbf{u}) d x_{1} d x_{2} .
$$

Using the elementary inequality $a^{2} \leq 2(a+b)^{2}+6 b^{2}$ we have

$$
\begin{aligned}
\int_{\Omega_{p}}\left(u_{1}^{2}+u_{2}^{2}\right) d x_{1} d x_{2} \leq & 2 \int_{\Omega_{p}}\left(\left(u_{1}+\frac{h}{2} \frac{\partial w}{\partial x_{1}}\right)^{2}+\left(u_{2}+\frac{h}{2} \frac{\partial w}{\partial x_{2}}\right)^{2}\right) d x_{1} d x_{2} \\
& +6 \int_{\Omega_{p}}\left(\left(\frac{h}{2} \frac{\partial w}{\partial x_{1}}\right)^{2}+\left(\frac{h}{2} \frac{\partial w}{\partial x_{2}}\right)^{2}\right) d x_{1} d x_{2} .
\end{aligned}
$$

In $\mathbf{H}^{2}\left(\Omega_{p}\right)$ we have

$$
\|w\|_{2, \Omega_{p}} \leq \mathrm{const}\left(\int_{\Omega_{p}}|w|^{2} d s+\langle w, w\rangle_{p}\right)^{1 / 2}
$$


where $w, w_{p}$ were already defined in Section 2 , and thus

$$
\int_{\Omega_{p}}\left(\left(\frac{h}{2} \frac{\partial w}{\partial x_{1}}\right)^{2}+\left(\frac{h}{2} \frac{\partial w}{\partial x_{2}}\right)^{2}\right) d x_{1} d x_{2} \leq \text { const } \int_{\Omega_{p}}|w|^{2} d s+\langle w, w\rangle_{p} .
$$

The combination of the last inequalities yields

$$
\begin{aligned}
& \left(\left\|u_{1}\right\|_{1, \Omega_{p}}^{2}+\left\|u_{2}\right\|_{1, \Omega_{p}}^{2}+\|w\|_{2, \Omega_{p}}^{2}\right)^{1 / 2} \\
& \left.\quad \leq \mathrm{const}\left(\int_{\Omega_{p}}\left(u_{1}+\frac{h}{2} \frac{\partial w}{\partial x_{1}}\right)^{2}+\left(u_{2}+\frac{h}{2} \frac{\partial w}{\partial x_{2}}\right)^{2}+w^{2}\right) d x_{1} d x_{2}+\langle\mathbf{u}, \mathbf{u}\rangle_{P}\right)^{1 / 2}
\end{aligned}
$$

which is the first part of the statement of the theorem. The inverse inequality is trivial and so the proof is completed.

LEMma 9. In $\mathcal{E}$ the norms $\|\mathbf{u}\|_{p b}$ and

$$
\left(\left\|u_{1}\right\|_{1, \Omega_{p}}^{2}+\left\|u_{2}\right\|_{1, \Omega_{p}}^{2}+\|w\|_{2, \Omega_{p}}^{2}+\left\|u_{1}\right\|_{1, V}^{2}+\left\|u_{2}\right\|_{1, V}^{2}+\|w\|_{1, V}^{2}\right)^{1 / 2}
$$

are equivalent.

Proof. We denote

$$
\alpha^{2}(\mathbf{u})=\left\|u_{1}\right\|_{1, \Omega_{p}}^{2}+\left\|u_{2}\right\|_{1, \Omega_{p}}^{2}+\|w\|_{2, \Omega_{p}}^{2}+\left\|u_{1}\right\|_{1, V}^{2}+\left\|u_{2}\right\|_{1, V}^{2}+\|w\|_{1, V}^{2} .
$$

The inequality $\|\mathbf{u}\|_{m p} \leq$ const $\alpha(\mathbf{u})$ is trivial. To prove the inverse inequality we first note that, due to the boundary condition (18), Korn's inequality, see [5], reads

$$
\left\|u_{1}\right\|_{1, V}^{2}+\left\|u_{2}\right\|_{1, V}^{2}+\|w\|_{1, V}^{2} \leq \operatorname{const}\langle\mathbf{u}, \mathbf{u}\rangle_{b}
$$

thus the restriction of $\mathbf{u} \in \mathcal{E}$ to $V$ is an element of the space $\left(\mathbf{H}^{1}(V)\right)^{3}$ and thus, by Theorem 3,

$$
\int_{\Omega_{p b}}\left(u_{1}^{2}+u_{2}^{2}+w^{2}\right) d x_{1} d x_{2} \leq \operatorname{const}\langle\mathbf{u}, \mathbf{u}\rangle_{b}
$$

Using the continuity conditions (20) and (21) we have therefore

$$
\begin{gathered}
\int_{\Omega_{p}}\left(\left(u_{1}+\frac{h}{2} \frac{\partial w}{\partial x_{1}}\right)^{2}+\left(u_{2}+\frac{h}{2} \frac{\partial w}{\partial x_{2}}\right)^{2}+w^{2}\right) d x_{1} d x_{2} \\
=\int_{\Omega_{p b}}\left(u_{1}^{2}+u_{2}^{2}+w^{2}\right) d x_{1} d x_{2} \leq \operatorname{const}\langle\mathbf{u}, \mathbf{u}\rangle_{b} .
\end{gathered}
$$

Adding the inequalities (22) and (23) we obtain

$$
\alpha^{2}(\mathbf{u}) \leq \text { const }\left(\int_{\Omega_{p}}\left(\left(u_{1}+\frac{h}{2} \frac{\partial w}{\partial x_{1}}\right)^{2}+\left(u_{2}+\frac{h}{2} \frac{\partial w}{\partial x_{2}}\right)^{2}+w^{2}\right) d x_{1} d x_{2}+\langle\mathbf{u}, \mathbf{u}\rangle_{p b}\right) .
$$

Usage of inequality (24) then completes the proof.

DEFINITION 10. $\mathbf{u} \in \mathcal{E}$ is called a generalized solution of the plate-body boundary value problem with geometrical constraints (18) if

$$
\langle\mathbf{u}, \delta \mathbf{u}\rangle_{p b}=\Lambda(\delta \mathbf{u}), \quad \text { for all } \delta \mathbf{u} \in \mathcal{E}
$$


Note that due to the conditions in (19) the term $\Lambda(\delta \mathbf{u})$ in this definition is well determined. Indeed, using standard integral inequalities we have

$$
\begin{aligned}
|\Lambda(\delta \mathbf{u})| \leq \sum_{j=1}^{3} & \left(\int_{V}\left|G_{i j}\right|^{6 / 5} d x_{1} d x_{2} d x_{3}\right)^{5 / 6}\left(\int_{V}\left|\delta u_{j}\right|^{6} d x_{1} d x_{2} d x_{3}\right)^{1 / 6} \\
& +\sum_{j=1}^{3}\left(\int_{\partial V}\left|g_{j}\right|^{4 / 3} d S\right)^{3 / 4}\left(\int_{\partial V}\left|\delta u_{j}\right|^{4} d S\right)^{1 / 4} \\
& +\sum_{j=1}^{2}\left(\int_{\Omega_{p}}\left|F_{j}\right|^{q} d x_{1} d x_{2}\right)^{1 / q}\left(\int_{\Omega_{p}}\left|\delta u_{j}\right|^{p} d x_{1} d x_{2}\right)^{1 / p} \\
& +\sum_{j=1}^{2}\left(\int_{\partial \Omega_{p}}\left|f_{j}\right|^{q} d s\right)^{1 / q}\left(\int_{\partial \Omega_{p}}\left|\delta u_{j}\right|^{p} d s\right)^{1 / p} \\
& +\left(\int_{\Omega_{p}}|F| d x_{1} d x_{2}\right)_{\Omega_{p}} \max _{\Omega_{p}}|\delta w| \\
& +\left(\int_{\partial \Omega_{p}}|f| d s\right)_{\partial \Omega_{p}}|\delta w|, \quad 1 / q+1 / p=1
\end{aligned}
$$

and thus using the embedding Theorem 3 we obtain

$$
\Lambda(\delta \mathbf{u}) \leq \text { const }\|\delta \mathbf{u}\|_{p b}
$$

Now we can formulate

TheOREM 11. Under the conditions (19), the three-dimensional body-plate boundary value problem has a unique generalized solution in the sense of Definition 10.

Proof. The inequality (26) means that the functional $\Lambda(\mathbf{u})$ is continuous in $E$, and we can apply the Riesz representation theorem to it:

$$
\Lambda(\delta \mathbf{u})=\left\langle\mathbf{u}_{0}, \delta \mathbf{u}\right\rangle_{p b}
$$

where $\mathbf{u}_{0} \in \mathcal{E}$ is uniquely defined. Then Eq. (25) takes the equivalent form

$$
\langle\mathbf{u}, \delta \mathbf{u}\rangle_{p b}=\left\langle\mathbf{u}_{0}, \delta \mathbf{u}\right\rangle_{p b} \quad \text { for all } \delta \mathbf{u} \in \mathcal{E}
$$

It is evident that the last equation has the unique solution $\mathbf{u}=\mathbf{u}_{0}$.

In order to obtain the classical formulation of the three-dimensional body-plate boundary value problem we suppose that $\mathbf{u}, \delta \mathbf{u} \in C_{2}$. We rewrite Eq. (25) in the following way:

$$
\langle\mathbf{u}, \delta \mathbf{u}\rangle_{P}+\langle\mathbf{u}, \delta \mathbf{u}\rangle_{b}-\Lambda(\delta \mathbf{u})=0
$$


Using integration by parts we have

$$
\begin{aligned}
\langle\mathbf{u}, \delta \mathbf{u}\rangle_{b}=- & -\int_{V} \frac{\partial \sigma_{i j}(\mathbf{u})}{\partial x_{j}} \delta u_{i} d x_{1} d x_{2} d x_{3}+\int_{\partial V} \sigma_{i j}(\mathbf{u}) \delta u_{i} n_{j} d S \\
\langle\mathbf{u}, \delta \mathbf{u}\rangle_{P}=- & \int_{\Omega_{p}} N_{\alpha \beta, \beta} \delta u_{\alpha} d x_{1} d x_{2}+\int_{\partial \Omega_{p}} N_{\alpha \beta} n_{\beta} \delta u_{\alpha} d s \\
& +\int_{\Omega_{p}} M_{\alpha \beta, \alpha \beta} \delta w d x_{1} d x_{2}+\int_{\partial \Omega_{p}}\left((\mathbf{M} \cdot \mathbf{n} \cdot \mathbf{n}) \frac{\partial \delta w}{\partial n}\right. \\
& \left.-\left(\mathbf{Q} \cdot \mathbf{n}+\frac{\partial M_{n \tau}}{\partial s}\right) \delta w\right) d s
\end{aligned}
$$

$\mathbf{n}=\left(n_{1}, n_{2}, n_{3}\right)$ being the unit outward normal vector to $\partial V$. We recall that on $\partial \Omega_{p}$ we have formally $\mathbf{n}=\left(n_{1}, n_{2}, 0\right)$. Then

$$
\int_{\partial V} \sigma_{i j}(\mathbf{u}) \delta u_{i} n_{j} d S=\int_{\partial V /\left(S \cup \Omega_{p b}\right)} \sigma_{i j}(\mathbf{u}) \delta u_{i} n_{j} d S+\int_{\Omega_{p b}} \sigma_{i 3}(\mathbf{u}) \delta u_{i} d x_{1} d x_{2} .
$$

Let us introduce the vector of displacements of the contact surface $\mathbf{u}_{p b}$ expressed in the terms of plate displacements,

$$
\mathbf{u}_{p b}\left(x_{1}, x_{2}\right)=\left(u_{1}\left(x_{1}, x_{2}\right)+\frac{h}{2} \frac{\partial w\left(x_{1}, x_{2}\right)}{\partial x_{1}}, u_{2}\left(x_{1}, x_{2}\right)+\frac{h}{2} \frac{\partial w\left(x_{1}, x_{2}\right)}{\partial x_{2}}, w\left(x_{1}, x_{2}\right)\right)
$$

Due to the continuity conditions (20) and (21), we formally obtain

$$
\int_{\Omega_{p b}} \sigma_{i 3}(\mathbf{u}) \delta u_{i} d x_{1} d x_{2}=\int_{\Omega_{p}} \sigma_{i 3}\left(\mathbf{u}_{p b}\right) \delta u_{i} d x_{1} d x_{2}
$$

and thus, after integrating by parts, we get

$$
\begin{aligned}
\int_{\Omega_{p b}} \sigma_{i 3}(\mathbf{u}) \delta u_{i} d x_{1} d x_{2}= & \int_{\Omega_{p}} \sigma_{\alpha 3}\left(\mathbf{u}_{p b}\right) \delta u_{\alpha} d x_{1} d x_{2}+\int_{\Omega_{p}} \sigma_{33}\left(\mathbf{u}_{p b}\right) \delta w d x_{1} d x_{2} \\
& -\frac{h}{2} \int_{\Omega_{p}} \frac{\partial \sigma_{\alpha 3}\left(\mathbf{u}_{p b}\right)}{\partial x_{\alpha}} \delta w d x_{1} d x_{2}+\frac{h}{2} \int_{\Omega_{p}} \sigma_{\alpha 3}\left(\mathbf{u}_{p b}\right) n_{\alpha} \delta w d s .
\end{aligned}
$$

A combination of Eqs. (30), (29), (28), and (27) yields

$-\int_{V}\left(\frac{\partial \sigma_{i j}(\mathbf{u})}{\partial x_{j}}+G_{i}\right) \delta u_{j} d x_{1} d x_{2} d x_{3}+\int_{\partial V /\left(S \cup \Omega_{p b}\right)}\left(\sigma_{i j}(\mathbf{u}) n_{j}-g_{i}\right) \delta u_{i} d S$

$-\int_{\Omega_{p}}\left(N_{\alpha \beta, \beta}+F_{\alpha}-\sigma_{\alpha 3}\left(\mathbf{u}_{p b}\right) \delta u_{\alpha} d x_{1} d x_{2}\right.$

$+\int_{\Omega_{p}}\left(M_{\alpha \beta, \alpha \beta}-F-\frac{h}{2} \frac{\partial \sigma_{\alpha 3}\left(\mathbf{u}_{p b}\right)}{\partial x_{\alpha}}+\sigma_{33}\left(\mathbf{u}_{p b}\right)\right) \delta w d x_{1} d x_{2}$

$+\int_{\partial \Omega_{p}}\left(N_{\alpha \beta} n_{\beta}-f_{\alpha}\right) \delta u_{\alpha} d s-\int_{\partial \Omega_{p}}\left(\mathbf{Q} \cdot \mathbf{n}+\frac{\partial M_{n \tau}}{\partial s}-\frac{h}{2} \sigma_{\alpha 3}\left(\mathbf{u}_{p b}\right) n_{\alpha}+f\right) \delta w d s$,

$+\int_{\partial \Omega_{p}}(\mathbf{M} \cdot \mathbf{n} \cdot \mathbf{n}) \frac{\partial \delta w}{\partial n} d s=0$ 
Applying standard tools of variational calculus we obtain

$$
\begin{aligned}
& \frac{\partial \sigma_{i j}(\mathbf{u})}{\partial x_{j}}+G_{i}=0 \text { in } V \\
& \sigma_{i j}(\mathbf{u}) n_{j}-g_{i}=0 \quad \text { on } \partial V /\left(S \cup \Omega_{p b}\right) \text {, } \\
& M_{\alpha \beta, \alpha \beta}+\sigma_{33}\left(\mathbf{u}_{p b}\right)-F-\frac{h}{2} \frac{\partial \sigma_{\alpha 3}\left(\mathbf{u}_{p b}\right)}{\partial x_{\alpha}}=0 \text { in } \Omega_{p} \text {, } \\
& N_{\alpha \beta, \alpha \beta}+F_{\alpha}-\sigma_{\alpha 3}\left(\mathbf{u}_{p b}\right)=0 \text { in } \Omega_{p}, \\
& \mathbf{M} \cdot \mathbf{n} \cdot \mathbf{n}=0 \quad \text { on } \partial \Omega_{p}, \\
& \mathbf{Q} \cdot \mathbf{n}+\frac{\partial M_{n \tau}}{\partial s}-\frac{h}{2} \sigma_{\alpha 3}\left(\mathbf{u}_{p b}\right) n_{\alpha}+f=0 \quad \text { on } \partial \Omega_{p}, \\
& N_{\alpha \beta} n_{\beta}-f_{\alpha}=0 \text { on } \partial \Omega_{p} .
\end{aligned}
$$

Adding the geometrical constraints (18), (20), and (21) we complete the classical statement of the problem under consideration.

For other sets of geometrical constraints, the classical statement can be obtained in a similar way.

Let us discuss the obtained relations. Equation (31) describing the state inside the body coincides with the equations of the theory of elasticity. All the other equations could be written in terms of boundary displacements of the body, and so they should be considered as the boundary conditions for the body. Nevertheless, (33), (34) describe the state of the plate and, neglecting the terms with $\sigma_{\alpha 3}$, coincide with the corresponding set of Kirchhoff equations as well as Eqs. (35)-(37) for the natural boundary condition; so the relations (33)-(37) look like a plane boundary value problem itself. Occurrence of the terms with $\sigma_{\alpha 3}$ does not allow consideration of this problem as independent, and so we have obtained a coupled problem of a new type with elliptic systems of equations given in domains of different dimensions, related to conditions of geometrical and mechanical nature.

4. Some concluding remarks. In the same way as above we could consider the problem of patching a plate and a three-dimensional body neglecting bending of the plate. Moreover, in a similar way, it is possible to state and investigate many different problems that fall into the same class of problems describing industrial constructions composed of elements with different mathematical models. We cite some of them:

- three-dimensional body-shell patched together;

- junction of two plates connected by a bar (using the classical model of a bar);

- two axisymmetrical shells connected by a ring (using the classical mathematical model of a ring).

The scheme for investigating them is the same and differs only in technical details.

We note that in another approach to a connected element, the second problem was considered by Bernardou [2].

For the same constructions we can consider eigenvalue problems using the same approach and, even dynamic problems transforming the approach correspondingly (see, for example, [4]). 
At last we note that the general form of the equation,

$$
\langle\mathbf{u}, \delta \mathbf{u}\rangle-L(\delta \mathbf{u})
$$

to describe the equilibrium of the systems under consideration falls into the class considered in [3] and so we can state that the application of finite-element methods in this class of problems is justified if the set of finite elements meets the approximation condition, namely every element of the corresponding energy space can be approximated in the energy norm by a finite-element solution with any degree of accuracy. Note that the sets of finite elements for each of the coupled bodies can be chosen according to their mathematical models and should be related in a certain way. For details we refer the reader to $[3]$.

\section{REFERENCES}

[1] R. A. Adams, Sobolev Spaces, Academic Press, New York, 1975

[2] M. Bernardou and S. Fayolle, Numerical junctions between plates, Computer Methods in Applied Mechanics and Engineering, vol. 74, 1989, pp. 307-326

[3] P. G. Ciarlet, The Finite Element Method for Elliptic Problems, North-Holland, Amsterdam, 1968

[4] L. P. Lebedev and I. I. Vorovich, On the Bubnov-Galerkin Method in the Nonlinear Theory of Vibrations of Viscoelastic Shells, Prikl. Mat. Meh., Vol. 37, 1973, pp. 1117-1124

[5] J. Necăs and I. Hlaváček, Mathematical Theory of Elastic and Elasto-Plastic Bodies, Elsevier Scientific Publishing, Amsterdam-Oxford-New York, 1981

[6] C. Truesdell and W. Noll, The Non-linear Field Theories of Mechanics, Handbuch der Physik III/3, Springer-Verlag, Berlin-Göttingen-Heildelberg, 1958 